\title{
Spatiotemporal Modulation of Central Neural Pathway Underlying Acupuncture Action: A Systematic Review
}

\author{
Lijun Bai ${ }^{1}$, Wei Qin ${ }^{2}$, Jimin Liang ${ }^{2}$, Jie Tian ${ }^{1,2, *}$ and Yijun Liu ${ }^{3}$
}

\author{
${ }^{1}$ Institute of Automation, Chinese Academy of Sciences, Beijing, 100190, China; ${ }^{2}$ Life Science Research Center, School \\ of Electronic Engineering, Xidian University, Xi'an, Shaanxi 710071, China; ${ }^{3}$ Departments of Psychiatry and Neurosci- \\ ence, McKnight Brain Institute, University of Florida, Gainesville, FL 32610, USA
}

\begin{abstract}
Acupuncture, an ancient therapeutic technique, is currently gaining popularity as an important modality of alternative and complementary medicine in the West world. Concurrently, scientific interests in exploring whether acupuncture is therapeutically effective are raised alongside those about the means by which it may operate. Modern neuroimaging techniques such as functional magnetic resonance imaging, positron emission tomography, electroencephalography, and magnetoencephalography provide a means to safely monitor brain activity in humans. In this review, we have summarized evidence derived from the neuroimaging studies and tried to elucidate the neurophysiological correlates of acupuncture. Previous investigations on the neural responses to acupuncture mainly focus on its acute effects and explore the correlation between the specific acupoints and cortical activations only in the spatial domain. However, abundant clinical reports and psychophysical analysis suggest the kinetics of acupuncture is longer acting as a function of time. Consequentially, an accurate interpretation of acupuncture actions depends on how effectively we can characterize the nature of temporal variations underlying neural activities, rather than simply detect the occurrence of such changes. This emerging picture indicates that both designing paradigms and statistical models involved in acupuncture studies should be applied with great care.
\end{abstract}

Keywords: Acupuncture, spatiotemporal response, brain correlates, neuroimaging studies.

\section{INTRODUCTION}

Acupuncture is an ancient East Asian healing modality that has been in use for more than 2000 years. Together with herbal medicine, it is regarded as one of the two most pivotal medical skills in East Asian medicines. In the last decades, acupuncture has gained great popularity as an alternative and complementary therapeutic intervention in the Western medicine [1]. In this process, the boundaries between East Asian medicines and biomedicine/science are porous, negotiated to connect different medical traditions. In spite of its public acceptance, increasing attentions are paid to explore the scientific explanation regarding the physiological mechanism of acupuncture.

Evidence from animal studies has demonstrated that acupuncture stimulation can facilitate the release of certain neuropeptides in the central nervous system (CNS), eliciting profound physiological effects and even activating selfhealing mechanisms [2-4]. Studies of electroacupuncture in rats revealed that both low-frequency and high-frequency stimulation could induce analgesia, but that there are differential effects of low- and high-frequency acupuncture on the types of endorphins released [5]. Peripheral acupuncture stimulation at deeper structures also activates various brain structures, such as the limbic, hypothalamic and brainstem neural nuclei [6]. Although animal research clearly supports a role for specific neural pathways underlying the action of acupuncture, it is difficult to interpret these studies in the

\footnotetext{
*Address correspondence to this author at the Institute of Automation, Chinese Academy of Sciences, P.O. Box 2728, Beijing, 100190, China; Tel: 86010-82618465, 86-029-88201842; Fax: 86-010-62527995;

E-mail: tian@iiee.org
}

context of more complex human experience, including the belief states, emotion and cognition changes. With improved resolution and sensitivity of the functional brain imaging techniques, such as positron emission tomography (PET) and functional magnetic resonance imaging (fMRI), better understanding of the mechanisms of acupuncture in human beings becomes available. The wide range of physical effects exerted by acupuncture and its purported efficacy for a compendium of clinical pathologies suggest that the brain may be responsible for transmitting the needle stimulus into signals aimed at maintaining homeostatic balance within and across functional subsystems [7-10]. In this review, we have systematically researched and reviewed the literature looking at the effect of acupuncture on brain activation and discussed how these findings contribute to current hypotheses of acupuncture action.

\section{NEUROIMAGING RESULTS OF ACUPUNCTURE IN HUMAN}

Human neuroimaging data on acupuncture at commonly used acupoints have demonstrated significant modulatory effects at various levels throughout the CNS. This includes endogenous antinociceptive limbic networks (cingulate cortex, insula, and hypothalamus) as well as higher-order cognitive and affective control centers within the prefrontal cortex and medial temporal lobe (amygdala and hippocampus) [1121]. Wu et al. found that stimulation at LI4 (located at the first dorsal intermetacarpal space with a depth of approximately $1 \mathrm{~cm}$ ) and ST36 (located four finger breadths below the lower margin of the patella and one finger breadth laterally from the anterior crest of the tibia) resulted in increases in signal intensity of the hypothalamus and nucleus accumbens, as well as decreases in the rostral part of the anterior 
cingulated cortex, amygdala, and hippocampus. However, these neural responses did not emerge in the control group [11]. This evidence supported the hypothesis that acupuncture can activate the structures of descending antinociceptive pathway and deactivate multiple limbic areas subserving affective dimension of pain. Similarly, Hui et al. reported that needle stimulation at ST36 induced a wider range of negative signal changes in the limbic-cerebellum system [16]. In addition, this decreased neural responses only occurred in the subjects experiencing the deqi sensations. In contrast, two subjects who experienced sharp pain instead of acupuncture sensation showed predominately increased instead of decreased signal intensity in the parahippocampus, anterior cingulate cortex (ACC), posterior cingulate cortex (PCC) and putamen. Given that deqi play a pivot role in the therapeutic effect of acupuncture [22, 23], this widely decreased limbic-cerebellum network may be one of the central characteristic involved in the action of acupuncture. Collectively, neuroimaging data strongly suggest that acupuncture modulates many distributed cortical and subcortical (i.e., brainstem, limbic, cerebellum) brain areas. These brain areas, supporting endogenous antinociceptive mechanism and part of the pain neuromatrix [24-26], may contribute to the therapeutic effect of acupuncture by shifting autonomic nervous system (ANS) balance, and altering the affective and cognitive dimensions of pain processing [27, 28].

\section{SUSTAINED EFFECTS OF ACUPUNCTURE AND ITS INFLUENCE ON fMRI RESULTS}

So far most neuroimaging studies mainly focus on the acute effects of acupuncture and adopt the general linear model (GLM) analysis [11-13, 17-19]. With this approach, a specific stimulus sequence (i.e., design matrix) is used to define an ideal hemodynamic response function (HRF), which is convolved with the actual hemodynamic response and produces predictors of the BOLD response [29]. In other words, changes in the BOLD signal, evoked by a particular psychphysiological event, are completely dependent on and specific to the experimental paradigm. For a block-designed fMRI paradigm, typical visual or motor tasks can detect the simultaneously activated nuclei successfully; the temporal changes in the BOLD signal as predicted by the GLM conform to the "on-off" specifications set by the experimenter. However, this may be not valid in the cases when limited or no prior temporal information is available, such as the study for testing the acute effects of acupuncture stimulus on the brain. On the other hand, abundant clinical reports have elucidated that acupuncture can provide relief beyond the time it is being performed [30]. Psychophysical analysis from Price et al. also suggests that the analgesic effects of acupuncture might actually peak long even after the needling session terminated [31]. Due to the sustained effect of acupuncture, the temporal aspects of the BOLD response to acupuncture may violate the assumptions of the block-designed GLM estimates (Fig. (1)) [32]. Thus, the magnitude of BOLD signal in rest period following the initial stimulation is unlikely to have returned to the initial (pre-stimulus) baseline level. Investigation from the independent component analysis (ICA) also provided direct evidence that interleaved resting epochs in the block-designed paradigm remain acupuncture-related changes [33]. Considering the temporal profile of acupuncture is slow to develop and resolve, both the design paradigm and statistical models should be promoted for which the actual effect of acupuncture can be appropriately studied.

An obstacle to multi-block design analysis is isolating the concurrent brain activity related to the acupuncture manipulation from the brain activity associated with the sustained effect resulting from the same stimulation. Qin et al. have proposed a newly experimental paradigm, namely the nonrepeated event-related fMRI (NRER-fMRI) design, to explore the prolonged effects of acupuncture on the restingstate brain networks [34]. Compared with the acupuncture at a non-acupoint (NAP), there was a higher level of correlations among the amygdala-associated networks following acupuncture at ST36. These areas mainly included the limbic/paralimbic areas (ACC and insula) and brainstem structures (periaqueductal gray, PAG) (Shown in Fig. (2)). Similarly, acupuncture, not the sham condition, can also induce the increased connectivity within both the default mode network and sensorimotor network [35], including the medial temporal lobe, PAG and supplementary motor area (SMA). They also reported that connectivity between the hippocampus and the DMN saliently correlated with parasympathetic output only following the acupuncture stimulation. This indicated that acupuncture may operate through the regulation of autonomic nervous system, which was consistent with increasing evidence for the involvement of autonomic efferent nerve activity underlying its analgesia effects [36-38]. Though preliminary, this phenomenon may reflect the specific neuro-modulatory effect of acupuncture on the resting brain $[39,40]$. In addition, a recent study has reported differences in resting-state brain functions of people with chronic pain in contrast with controls, and the authors proposed that this difference in resting-state brain activity might reflect the cognitive and affective complications of chronic pain [41]. Along these lines, exploration of the alternating interplay between the external acupuncture intervention and the organization of resting-state networks, can not only help us better understand the long-term effects of pain on the brain, but also the potential benefits of acupuncture in pain treatments. However, this hypothesis needs further investigations in the altered and/or dysfunctional brain networks such as those in patients with chronic pain.

If acupuncture is a slow-acting agent, the temporal information involved may help further enclose its specific mechanism on brain modulation. One pioneer study developed a novel approach (defining separate models) to evaluate the dynamic signal changes between the baseline activity (prior to stimulation) and the neural activity in sequential epochs within the multi-block design of acupuncture [32]. Results presented that acupuncture at ST 36 can induce significantly time-varied response patterns in the wider extent of the limbic-cerebellar and brainstem structures (Fig. (1)). These notable BOLD signal changes share a common feature that the identified voxels, containing a population of neurons, coded the temporal dimension [42-44]. Some regions only responded to the initial administration of acupuncture (PCC), and others may ramp up or taper off across the whole session (PAG; rostral ventromedial medulla, RVM). Yet other brain areas showed sustained responses (Insula), and continuously exerted controlling and coordinated influences throughout the scanning. Even in the same regions (limbiccerebellar system), there were distinct temporal responses, 


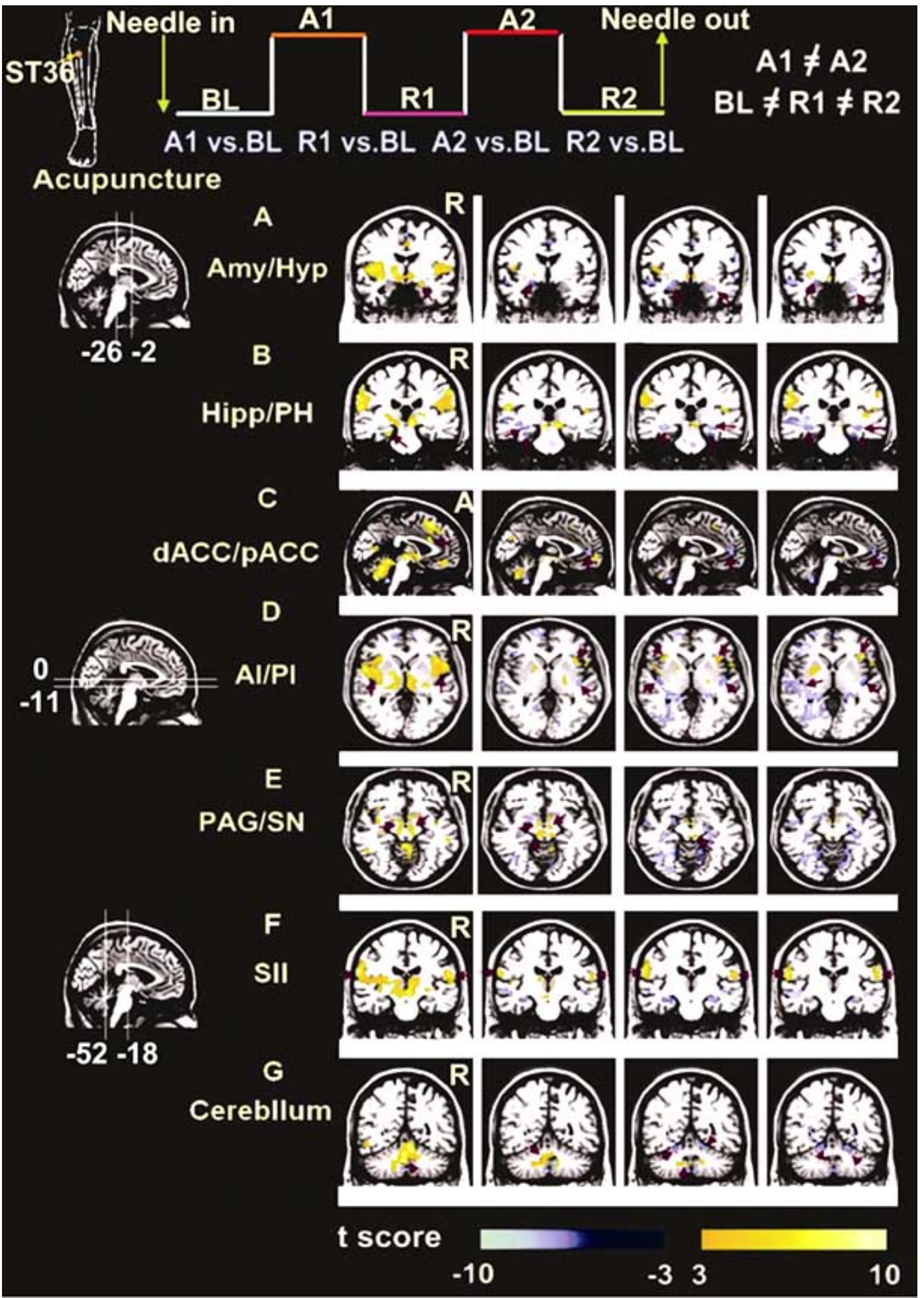

Fig. (1). Activity patterns of representative areas under different epochs of multi-block following acupuncture stimulation on ST36 (P $<0.005$, uncorrected, cluster size $>3$ voxels). The kinetics of acupuncture was complex and longer acting as a function of time, rather than a simple variation in the form of the "on-off" variations predicated by the block-based GLM analysis. Corresponding t values of representative regions under different periods were also indicated (Error bars SEM). Row A and B: the amygdala (Amy), hippocampus (Hipp), and parahippocampus ( $\mathrm{PH}$ ) presented weakly positive responses in the first stimulation A1, but decreased to the belowbaseline level thereafter. Row C: early positive signal response was particularly located in the dorsal part of the ACC, and negative in the pregenual part in sequential conditions. Row D and F: the insula (both anterior and posterior part, AI and PI) and secondary somatosensory cortex (SII) indicated relatively persistent increased responses during the whole trial. Row A and E: episodic responses were primarily distributed in the hypothalamus (Hyp), and brainstem structures (periaqueductal gray PAG; substania nigra, SN). As shown in the PAG, the greatest positive activity emerged in the R1, plateaued in the A2, but showed no significant activity in both A1 and R2 conditions. Row G: The BOLD responses in the cerebellum (anterior part - culmen and posterior part - declive) were positive predominantly, and negative afterwards (adapted from ref. [32]). 


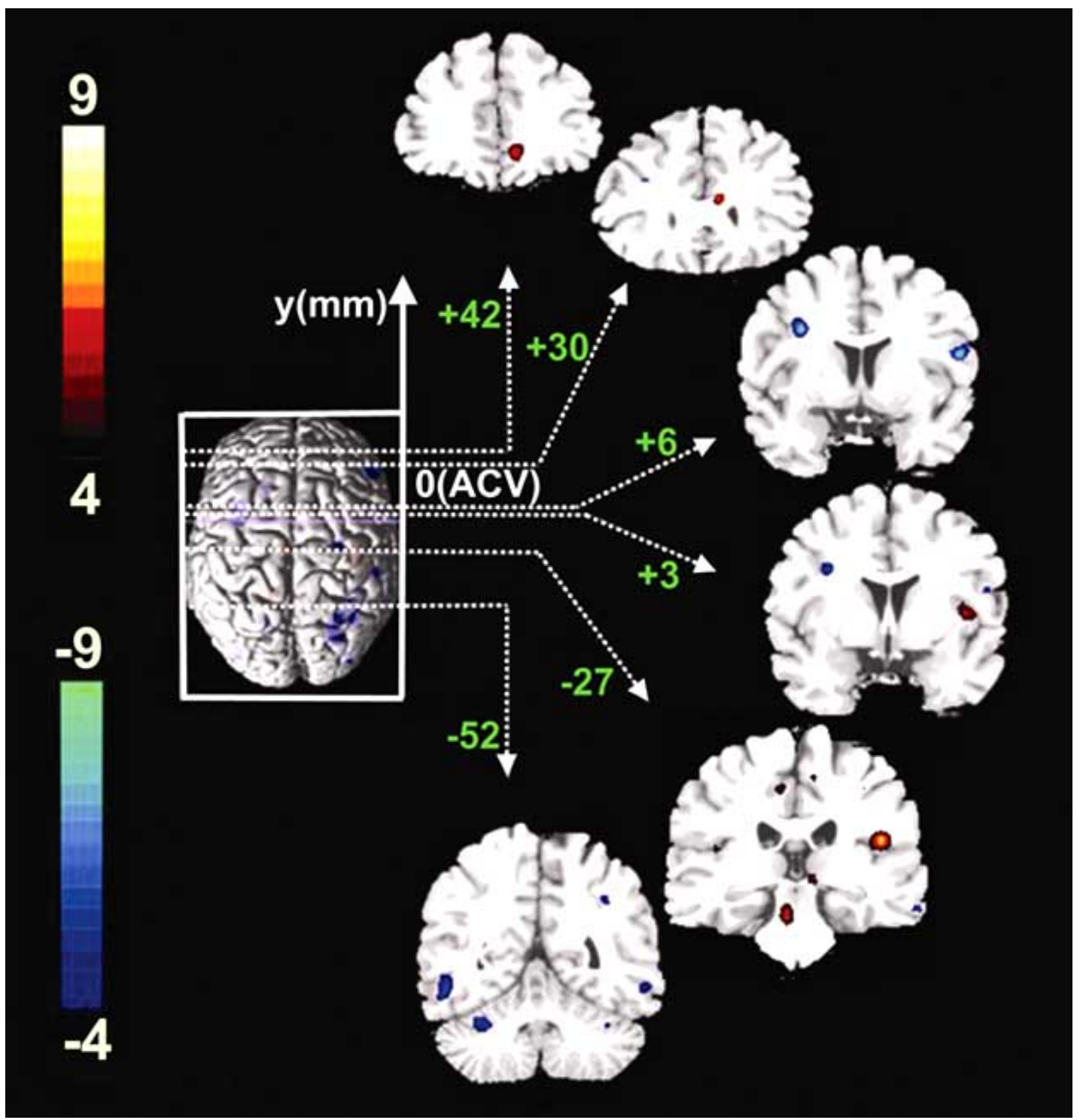

Fig. (2). Acupuncture enhanced the correlations of the amygdala with the insula, anterior cingulate cortex and periaqueductal gray during the post-stimulus resting brain, while sham evoked the increased correlations of the amygdala with the secondary somatosensory cortex and cerebellum. ACV: anterior commissure verticalization (adapted from ref. 34).

reflecting a significant modulation of response directions during different stages of the acupuncture. Limbic and brainstem areas have been indicated to support endogenous antinociceptive mechanisms and are part of the "pain neuromatrix". Signal attenuation in the amygdala was also observed to be correlated with the elevation of pain threshold in subjects [17]. In addition, evidence from animal studies suggests that acupuncture analgesia may be largely supported by endogenous opioidergic and/or monoaminergic "antinociceptive" networks [6]. One of these mechanisms, by which the PAG modulates the ascending sensory responses, involves releasing endogenous opioids to exert its anti-nociceptive effect through the opioid synapses of the RVM system [45]. The RVM has distinct but anatomically overlapping populations of modulatory neurons: off cells that inhibit and on cells that facilitate nociceptive transmission [46, 47]. This could partially explain the RVM showed more complex (bidirectional) activity patterns as reported by [32]. Cells throughout this network could always fire simultaneously, implicating the PAG-RVM network functions as a unit and exerting discrete control, globally rather than topographically, over dorsal horn pain transmission neurons. In addition, PAG activity may be triggered or facilitated by "topdown" pain signaling from higher centers including the insula and ACC [48-51]. These areas, along with limbic regions including the hippocampus and amygdala, are involved in the descending antinociceptive pathway. Taken together, the action underlying the acupuncture may alter the pain perception by inhibiting the antinociceptive action in the affective pathway while mobilizing the descending mechanisms by controlling the transmission of nociceptive signals to the brain.

\section{NEURAL CORRELATES OF ACUPOINT SPECIFIC- ITY FROM SPATIOTEMPORAL DOMAIN}

Acupoint specificity lies at the core of traditional acupuncture theory. The clinical effectiveness of acupuncture per se is said to depend upon the specific placement of the needles at designated acupuncture points [8]. However, the evidence supporting the specificity of acupuncture modula- 
tory effects splits in both the positive and negative side. Cho was one of pioneers to find that the visual cortex can be induced by peripheral acupuncture at visual-associated acupoints in the same way as it was evoked by a direct visual stimulus [52]. This neural response of the visual cortex did not emerge after acupuncture at a non-acupoint. This specificity was entirely consistent with a Traditional Chinese Medicine (TCM) interpretation of the acupuncture point's function. Further investigation by others (Li et al., 2003; Lee et al., 2002) also supported that acupuncture given at traditional "vision-related" acupoints can elicit activity selectively within the visual (occipital) cortex [53-56]. However, such acupoint specificity has been controversial and difficult to replicate in other fMRI studies [57, 58]. Investigations from Gareus et al. throwed some doubt on the specificity of visual-related acupoints [57]. In a recent study, Kong and their colleagues have found that acupuncture at the visualrelated acupoint and non-acupoint can both evoke negative signal changes of the visual cortices [59]. On balance, however, it doesn't seem that acupuncture is devoid of specific effects. Given that previous studies have only investigated the spatial distribution of neural response to acupuncture at specific acupoints, the temporal information involved may be ignored.

As aforementioned, the kinetics of acupuncture is inherently complex and time-dependent. An accurate interpretation of acupuncture actions may depend on how effectively we can characterize the nature of temporal variations underlying neural activities that give rise to hemodynamic responses, rather than simply detect the occurrence of such changes. It is speculated that differences between verum and sham acupuncture may also occur in the temporal domain, which may be resolved by neuroimaging modalities such as MEG. One preliminary data indicate that electroacupuncture and sham acupuncture (noninsertive tapping) can both evoke responses in the primary somatosensory cortex (SI) [60, 61]. However, initial response to sham acupuncture peaks at longer post-stimulus latencies ( $\sim 35$ milliseconds) than verum EA ( 20 milliseconds). It may possibly be as a result of temporal dispersion derived from different stimulation mode (mechanical versus electrical stimuli). While the subtle temporal differences are identified, the exact meaning of this specific response is yet unresolved. Further work is certainly required to ascertain whether modulation of these areas is related to the specificity of acupuncture effects.

\section{FURTHER DIRECTIONS FOR ACUPUNCTURE NEUROIMAGING RESEARCH}

The majority of neuroimaging researchers have suffered from multiple methodological challenges in order to sufficiently address the complexities of the therapeutic mechanisms of acupuncture. Variability in needling technique, deqi sensations, design paradigm, differences in neruoimaging hardware and software, as well as data post-processing methods [62, 63], may all account for many of the reported differences in brain response. Therefore, it may be helpful to define a standardized reporting system to describe details of acupuncture manipulations [64]. Furthermore, it is not known which aspects of the acupuncture treatment, such as the mode of stimulation or location of the acupuncture point, are specific to produce these physiological effects. The con- trol group is therefore designed and plays a central role in the research model. Despite several control modalities are available, it is still controversial which one is appropriate. One approach is to apply the retractable nonpenetrating sham needle [65], which gives the impression of skin penetration without piercing the skin. It mainly served as a control for non-specific cognitive factors (e.g. expectation), whereas it may also lead to the subjects' bias toward the stimulation. Another control modality is the sham acupuncture, which is performed on a nearby non-acupoint with needle depth, stimulation intensity, and manipulation method all identical to those used in the verum acupuncture. Although it is proved to be far from inactive and does in fact have a physiological effect [66-69], careful design and execution procedures can make it useful to assess the neural specificity of acupuncture with respect to different locations. Considering acupuncture is inherently multifaceted, the decision as to which control should be used will ultimately depend on the particular question that the research model plans to answer. It is thus important for researchers to clearly explain the target questions and select the appropriate control to match the purpose of the study [70].

Previous acupuncture studies have generally adopted the multi-block design paradigm with repeated stimuli during a relatively short-term time span. Since acupuncture-related neural responses can be long-lasting and not return to the baseline level immediately after the stimuli terminated, the "on-off" specifications set by block design may be violated; further analysis may be susceptible to errors of statistical significance [32]. In block designs, it is also difficult to disentangle the concurrent brain activity related to the needling manipulation from the brain activity associated with its sustained effect resulting from the same stimulation. Therefore, the non-repeated event-related design paradigm may be more optimal in acupuncture studies [34]. Furthermore, the model-based analysis (GLM) also becomes impractical when the precise timing and duration of acupuncture cannot be specified a priori. In other words, the depiction of dynamic on-going acupuncture effects would be obtained in the absence of any assumption concerning the shape of the hemodynamic response. For this purpose, the data-driven analysis, free of any hypothesis about the temporal profile of acupuncture-related changes, can be a more optimal choice instead.

\section{CONCLUSION}

This paper briefly researched and reviewed the literature regarding the neurophysiologic mechanisms of acupuncture as measured by modern functional neuroimaging techniques. Future studies evaluating both central and peripheral effects of needle stimulation, in a well-controlled disease model, may ultimately help to determine which the neurological substrates of acupuncture contribute to its specific clinical effects. Concurrent physiological measurements (e.g. electrocardiography, pupillometry, electro-dermal activity) should also be adopted in conjunction with neuroimaging to help correlate acupuncture-related neural response with ANS functions. Hopefully, as modern neuroimaging research rigorous and enthusiastically explores this ancient therapy, horizons will be broadened and help improve acupuncture's potential clinical applications in expanded areas. 


\section{ACKNOWLEDGEMENTS}

This work is supported by Changjiang Scholars and Innovative Research Team in University (PCSIRT) under Grant No.IRT0645, Chair Professors of Cheung Kong Scholars Program, CAS Hundred Talents Program, the Joint Research Fund for Overseas Chinese Young Scholars under Grant No.30528027, the National Natural Science Foundation of China under Grant No. 30873462, 90209008, 30870685, 30672690, 30600151, 60532050, 60621001, the Beijing Natural Science Fund under Grant No. 4071003, the Project for the National Key Basic Research and Development Program (973) under Grant No.2006CB705700, and 863 program under Grant No. 2008AA01Z411.

\section{REFERENCES}

[1] NIH. NIH consensus development panel on acupuncture. J Am Med Assoc 1998; 280: 1518-29.

[2] Han JS. Acupuncture: neuropeptide release produced by electrical stimulation of different frequencies. Trends Neurosci 2004; 26: 1722.

[3] Xing GG, Liu FY, Qu XX, et al. Long-term synaptic plasticity in the spinal dorsal horn and its modulation by electroacupuncture in rats with neuropathic pain. J Pharmacol Exp Ther 2007; 321: 104653.

[4] Wang Q, Mao LM, Han JS. Characterization of inhibition of spinal nociceptive reflex by stimulation of the arcuate nucleus of the hypothalamus in the pentobarbital-anesthetized rat. Pain 1990; 41: $101-8$

[5] Guo HF, Tian J, Wang X, Fang Y, Hou Y, Han J. Brain substrates activated by electroacupuncture (EA) of different frequencies (II): Role of Fos/Jun proteins in EA induced transcription of preproenkephalin and pre-S-126 SHEN prodynorphin genes. Brain Res Mol Brain Res 1996; 43: 167-73.

[6] Pomeranz B. Acupuncture analgesia: basic research. In: Stux G, Hammerschlag R, Eds. Clinical acupuncture: scientific basis. Berlin: Springer 2000; pp. 1-28.

[7] Mann F. Reinventing Acupuncture: A new concept of ancient medicine. Great Britain: Biddles Ltd. 1992.

[8] Kaptchuk TJ. Acupuncture: theory, efficacy, and practice. Ann Intern Med 2002; 136: 374-83.

[9] White A, Foster NE, Cummings M, Barlas P. Acupuncture treatment for chronic knee pain: a systematic review. Rheumatology 2007; 46: 384-90

[10] Jeanette E, Brian B, Victoria AH, et al. Is acupuncture effective for the treatment of chronic pain? A systematic review. Pain 2000; 86: 217-25.

[11] Wu MT, Hsieh JC, Xiong J, et al. Central nervous pathway for acupuncture stimulation: localization of processing with functional MR imaging of the brain-Preliminary experience. Radiology 1999; 212: $133-41$.

[12] Wu MT, Sheen JM, Chuang KH, et al. Neuronal specificity of acupuncture response: a fMRI study with electroacupuncture. NeuroImage 2002; 16: 1028-37.

[13] Fang JL, Krings T, Weidemann J, Meister IG, Thron A. Functional MRI in healthy subjects during acupuncture: different effects of needle rotation in real and false acupoints. Neuroradiology 2004; 46: 359-62.

[14] Pariente J, White P, Frackowiak RSJ, Lewith G. Expectancy and belief modulate the neuronal substrates of pain treated by acupuncture. Neuroimage 2005; 25: 1161-7.

[15] Hui KK, Liu J, Makris N, et al. Acupuncture modulates the limbic system and subcortical gray structures of the human brain: evidence from fMRI studies in normal subjects. Hum Brain Mapp 2000; 9: 13-25.

[16] Hui KK, Liu J, Marina O, et al. The integrated response of the human cerebro-cerebellar and limbic systems to acupuncture stimulation at ST 36 as evidenced by fMRI. Neuro Image 2005; 27: 47996

[17] Zhang WT, Jin Z, Cui GH, et al. Relations between brain network activation and analgesic effect induced by low vs. high frequency electrical acupoint stimulation in different subjects: a functional magnetic resonance imaging study. Brain Res 2003; 982: 168-78.

[18] Liu WC, Feldman SC, Cook DB, et al. 2004. fMRI study of acupuncture-induced periaqueductal gray activity in humans. Neuro Report 2004; 15: 1937-40.

[19] Yoo SS, The EK, Blinder RA, Jolesz FA. Modulation of cerebellar activities by acupuncture stimulation: evidence from fMRI study. Neuro Image 2004; 22: 932-40.

[20] Napadow V, Liu J, Li M, et al. Somatosensory cortical plasticity in carpal tunnel syndrome treated by acupuncture. Hum Brain Map 2007; 30: 38-46.

[21] Kong J, Ma L, Gollub RL, et al. A pilot study of functional magnetic resonance imaging of the brain during manual and electroacupuncture stimulation of acupuncture point (LI-4 Hegu) in normal subjects reveals differential brain activation between methods. J Altern Complement Med 2002; 8: 411-9.

[22] Takeda W, Wessel J. Acupuncture for the treatment of pain of osteoarthritic knees. Arthe Care Res 1994; 7: 118-22.

[23] Witt C, Brinkhaus B, Jena S, et al. Acupuncture in patients with osteoarthritis of the knee: a randomised trial. Lancet $2005 ; 366$ 136-43.

[24] Casey KL. Forebrain mechanisms of nociception and pain: analysis through imaging. Proc Natl Acad Sci USA 1999; 96: 7668-74.

[25] Fields HL, Basbaum AI. Central nervous system mechanisms of pain modulation, Textbook of Pain. Wall PD, Melzack R, Eds. Edinburgh, Churchill Livingstone 1999; PP. 309-29.

[26] Hofbauer RK, Rainville P, Duncan GH, Bushnell MC. Cortical representation of the sensory dimension of pain. J Neurophysiol 2001; 86: 402-11.

[27] Peets JM, Pomeranz B. CXBK mice deficient in opiate receptors show poor electroacupuncture analgesia. Nature 1978; 273: 675-6.

[28] Clement-Jones V, McLoughlin L, Tomlin S, et al. Increased betaendorphin but not met-enkephalin levels in human cerebrospinal fluid after acupuncture for recurrent pain. Lancet 1980; 2: 946-9.

[29] Worsley KJ, Friston KJ. Analysis of fMRI time-series revisitedagain. Neuroimage 1995; 2: 173-81.

[30] Beijing S. Nanjing colleges of Traditional Chinese Medicine. Essentials of Chinese acupuncture. Beijing: Foreign Language Press 1980.

[31] Price DD, Rafii A, Watkins LR, Buckingham B. A psychophysical analysis of acupuncture analgesia. Pain 1984; 19: 27-42.

[32] Bai LJ, Qin W, Tian J, et al. Time-varied characteristics of acupuncture effects in fMRI studies. Hum Brain Mapp 2009; DOI: 10.1002/hbm.20769.

[33] Zhang Y, Qin W, Liu P, et al. An fMRI study of acupuncture using independent component analysis. Neurosci Lett 2009; 449: 6-9.

[34] Qin W, Tian J, Bai LJ, et al. fMRI connectivity analysis of acupuncture effects on an amygdala-associated brain network. Mol Pain 2008; 4: 55

[35] Dhond RP, Yeh C, Park K, Kettner N, Napadow V. Acupuncture modulates resting state connectivity in default and sensorimotor brain networks. Pain 2008; 136: 407-18.

[36] Haker E, Egekvist H, Bjerring P. Effect of sensory stimulation (acupuncture) on sympathetic and parasympathetic activities in healthy subjects. J Auton Nerv Syst 2000; 79: 52-9.

[37] Hsu CC, Weng CS, Sun MF, Shyu LY, Hu WC, Chang YH. Evaluation of scalp and auricular acupuncture on EEG, HRV, and PRV. Am J Chin Med 2007; 35: 219-30.

[38] Sakai S, Hori E, Umeno K, Kitabayashi N, Ono T, Nishijo H. Specific acupuncture sensation correlates with EEGs and autonomic changes in human subjects. Auton Neurosci 2007; 133: 158-69.

[39] Bai LJ, Qin W, Tian J, et al. Acupuncture modulates spontaneous activities in the anticorrelated resting brain networks. Brain Res 2009; 1279(7): 37-49.

[40] Bai LJ, Qin W, Tian J, et al. Detection of dynamic brain networks modulated by acupuncture using a graph theory model. Prog Nat Sci 2009; 19: 827-36.

[41] Baliki MN, Geha PY, Apkarian AV, Chialvo DR. Beyond feeling: chronic pain hurts the brain, disrupting the default-mode network dynamics. J Neurosci 2008; 28: 1398-403.

[42] Faingold CL, Randall ME. Pontine reticular formation neurons exhibit a premature and precipitous increase in acoustic responses prior to audiogenic seizures in genetically epilepsy-prone rats. Brain Res 1995; 704: 218-26. 
[43] Petrovic P, Petersson KM, Hansson P, Ingvar M. Brainstem involvement in the initial response to pain. Neuroimage 2004; 22 : 995-1005.

[44] Quirk GJ, Mueller D. Neural mechanisms of extinction learning and retrieval. Neuropsychopharmacology 2007; 33: 56-72.

[45] Willis WD. Central nervous system mechanisms for pain modulation. Appl Neurophyziol 1985; 48: 153-65.

[46] Haws CM, Williamson AM, Fields HL. Putative nociceptive modulatory neurons in the dorsolateral pontomesencephalic reticular formation. Brain Res 1989; 483: 272-82.

[47] Tortorici V, Vanegas H. Putative role of medullary off- and oncells in the antinociception produced by dipyrone (metamizol) administered systemically or microinjected into PAG. Pain 1994; 57 : 197-205.

[48] Fields HL, Heinricher MM, Mason P. Neurotransmitters in nociceptive modulatory circuits. Annu Rev Neurosci 1991; 14: 219-45.

[49] Millan MJ. Descending control of pain. Prog Neurobiol 2002; 66: 355-474.

[50] Porreca F, Ossipov MH, Gebhart GF. Chronic pain and medullary descending facilitation. Trends Neurosci 2002; 25: 319-25.

[51] Urban MO, Gebhart GF. Supraspinal contributions to hyperalgesia. Proc Nat Acad Sci USA 1999; 96: 7687-92.

[52] Cho Z, Chung S, Jones J, et al. New findings of the correlation between acupoints and corresponding brain cortices using functional MRI. Proc Natl Acad Sci USA 1998; 95: 2670-3.

[53] Li G, Cheung RTF, Ma QY, Yang ES. Visual cortical activations on fMRI upon stimulation of the vision-implicated acupoints. Brain Imaging 2003; 14: 669-73.

[54] Lee H, Park HJ, Kim SA, et al. Acupuncture stimulation of the vision-related acupoint (B1-67) increases c-Fos expression in the visual cortex of binocularly deprived rat pups. Am J Chin Med 2002; 30: 379-85.

[55] Li G, Raymond TF, Cheung CA, Ma QY, Yang ES. Visual cortical activations on fMRI upon stimulation of the vision-implicated acupoints. NeuroReport 2003; 14: 669-73.

[56] Christian M, Siedentopf SM, Golaszewski FM, Mottaghy CC, Ruff SF, Andreas S. Functional magnetic resonance imaging detects activation of the visual association cortex during laser acupuncture of the foot in humans. Neurosci Lett 2002; 327: 53-6.

[57] Gareus IK, Lacour M, Schulte AC, Hennig J. Is there a BOLD response of the visual cortex on stimulation of the vision-related acupoint GB 37? J Magn Reson Imaging 2002; 15: 227-32.
[58] Parrish TB, Schaeffer A, Catanese M, Rogel MJ. Functional magnetic resonance imaging of real and sham acupuncture: noninvasively measuring cortical activation from acupuncture. IEEE Eng Med Biol Mag 2005; 24: 35-40.

[59] Kong J, Kaptchuk TJ, Webb JM, et al. Functional neuroanatomical investigation of vision-related acupuncture point specificity-A multisession fMRI study. Hum Brain Mapp 2009; 30: 38-46.

[60] Dhond RP, Witzel T, Yeh C, et al. Spatiotemporal mapping the neural correlates of acupuncture. 13th Ann Org Hum Brain Map Conf, Chicago 2007.

[61] Dhond RP, Witzel T, Yeh C, et al. Mapping the neural correlates of acupuncture with magnetoencephalography. Baltimore: Society for Acupuncture Research 2007.

[62] Smith SM, Beckmann CF, Ramnani N, et al. Variability n fMRI: a re-examination of inter-session differences. Hum Brain Mapp 2005; 24: 248-57.

[63] Kong J, Randy LG, Webb JM, Kong JT, Vangel MG, Kwong K. Test-retest study of fMRI signal change evoked by electroacupuncture stimulation. Neuro Image 2007; 34: 1171-81.

[64] MacPherson H, White A, Cummings M, Jobst K, Rose K, Niemtzow R. Standards for reporting interventions in controlled trials of acupuncture: The STRICTA recommendations. J Altern Complement Med 2002; 8: 85-9.

[65] Streitberger K, Kleinhenz J. Introducing a placebo needle into acupuncture research. Lancet 1998; 352: 364-5.

[66] Le BD, Villanueva L, Willer J, Bouhassira D. Diffuse noxious inhibitory controls (DNIC) in animals and man. Acupuncture Med 1991; 9: 47-56.

[67] Staebler FE, Wheeler J, Young J, et al. Why research into traditional Chinese acupuncture has proved difficult. Strategies of the Council for Acupuncture, UK, to overcome the problem. Complementary Ther Med 1994; 2: 86-92.

[68] Ernst E, White AR. A review of problems in clinical acupuncture research. Am J Chin Med 1997; 25: 3-11.

[69] Vincent C, Lewith G. Placebo controls for acupuncture studies. J R Soc Med 1995; 88, 199-202.

[70] Sherman KJ, Lao LX, MacPherson H, Lewith G, Hopwood V, Kawakita K. Matching acupuncture clinical study designs to research questions. Clin Acupunct Orient Med 2002: 3: 12-5. 